\title{
Reply to Laprie A. et al
}

Johannes A. Langendijk, Esther G.C. Troost, Karin Haustermans, Cai Grau.

We thank our colleagues Laprie et al. for their valuable comments on the paper reporting on the activities explored by the European Proton Therapy Network and we are glad to notice that they confirm the need and importance of this initiative.

They expressed their concern that DICOM-RT data are not (yet) mandatory in the data collection program. It should be noted that EPTN is just in the initial phase of its existence and in this phase the main objective is to set up a programme, which can be easily implemented by the participating and future centres. First, our highest priority is to set up a pan-European programme in which all proton therapy centres can and will participate. Consequently, centres that decide to participate should commit to provide all level I (mandatory) data. In other words, we prefer to enable participation of all centres with high compliance for an initially limited number of data rather than participation of only a limited number of centres with extensive data. Second, the EPTN Generic Dataset as presented in the paper should not be regarded as final.

As soon as the level I assessments have been implemented successfully, the plan is indeed to start with collection of DICOM-RT data as well as to upgrade these data collections from level II to level I, which will indeed allow for more extensive quality assurance programs. We believe that only by doing so, sufficiently large data sets for the selection of patient cohorts benefitting from proton therapy can be collected.

It should be stressed that we only presented a first version of the proposed Generic Data Set, which will be supplemented with various more extensive Tumour-specific Data Sets for different tumour sites, including CNS, head and neck, breast, lung, oesophagus and prostate. For the paediatric patients, EPTN has a strong preference to link to the ongoing prospective data registry recently launched in the United States, to enable posthoc data linking and joint analyses in transatlantic projects. 